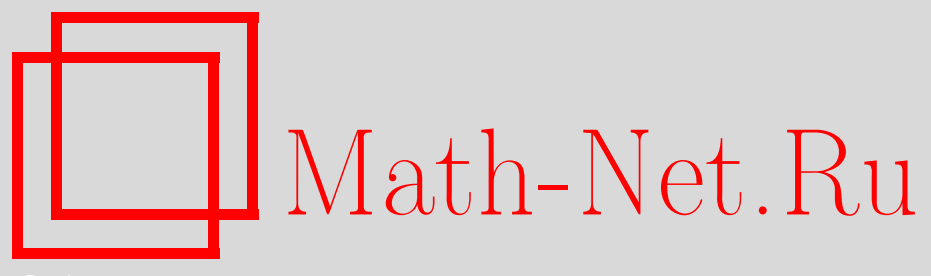

T. Р. Азаматов, Эффективные оценки для обобщенных глобальных соотношений, УМН, 2007, том 62, выпуск $5,145-146$

DOI: https://doi.org/10.4213/rm6427

Использование Общероссийского математического портала Math-Net.Ru подразумевает, что вы прочитали и согласны с пользовательским соглашением http://www.mathnet.ru/rus/agreement

Параметры загрузки:

IP : 35.173 .219 .12

26 апреля 2023 г., 12:39:58

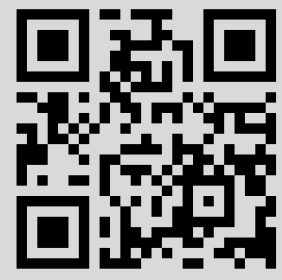




\section{Эффективные оценки для обобщенных глобальных соотношений}

\section{T. Р. Азаматов}

В работе использована $p$-адическая модификация метода Зигеля-Шидловского [1].

Пусть $\mathbb{K}$ - алгебраическое числовое поле конечной степени $\kappa$ над полем $\mathbb{Q}, V-$ множество всех нормирований поля $\mathbb{K}$. Для $v \in V$ обозначим $\mathbb{K}_{v}$ пополнение поля $\mathbb{K}$ по этому нормированию. Если $p$ - простое число, $v \mid p$, то считаем, что $|p|_{v}=p^{-\kappa_{v} / \kappa}$, где $\kappa_{v}=\left[\mathbb{K}_{v}: \mathbb{Q}_{p}\right]$.

В 1981 г. Э. Бомбиери в работе [2] ввел понятие глобального соотношения. Пусть $P\left(y_{1}, \ldots, y_{m}\right)$ - многочлен с коэффициентами из $\mathbb{K}$, степенные ряды $f_{1}(z), \ldots, f_{m}(z)$ имеют коэффициенты из $\mathbb{K}, \xi \in \mathbb{K}$. Соотношение $P\left(f_{1}(\xi), \ldots, f_{m}(\xi)\right)=0$ называется глобалъным, если оно выполняется во всех тех полях $\mathbb{K}_{v}$, где сходятся ряды $f_{1}(\xi), \ldots, f_{m}(\xi)$. В [2] это понятие было исследовано для так называемых $G$-функций.

В 1990 г. В.Г. Чирский [3] рассмотрел $F$-ряды, естественно дополняющие $E$ - и $G$-функции Зигеля (определение $E$ - и $G$-функций дано в [1]). Ряд $f(z)=\sum_{n=0}^{\infty} a_{n} n ! z^{n}$ принадлежит классу $F\left(\mathbb{K}, c_{1}, c_{2}, c_{3}, q\right)$, если: 1) $\left.\left|\overline{a_{n}}\right|=O\left(\exp \left(c_{1} n\right)\right), n \rightarrow \infty ; 2\right)$ существует последовательность натуральных чисел $d_{n}=q^{n} d_{0, n}$, где $q \in \mathbb{N}$, такая, что для всех $n$ и $k=0,1, \ldots, n$ числа $d_{n} a_{k} \in \mathbb{Z}_{\mathbb{K}}$. При этом натуральные числа $d_{0, n}$ делятся только на простые числа $p$, не превосходящие $c_{2} n$, и $\operatorname{ord}_{p} d_{0, n} \leqslant c_{3}\left(\log _{p} n+n / p^{2}\right)$. Пусть $c_{1}^{*}=c_{1}+\ln 2, c_{2}^{*}=\max \left(c_{2}, 1\right), c_{3}^{*}=\left(c_{3}+1\right) d, q^{*}=q^{1+[\ln d]}$.

В работах [3], [4] с помощью $p$-адической модификации метода Зигеля-Шидловского получен критерий отсутствия глобальных соотношений для $F$-рядов, удовлетворяющих дифференциальным уравнениям. В 2004 г. в статье Д. Бертрана, В. Г. Чирского, Й. Йеббоу [5] установлен критерий, в котором входящие в него оценки полностью эффективны.

В 1970 г. А. И. Галочкин [6] опубликовал теорему об алгебраической независимости значений $E$-функций в трансцендентных точках, допускающих достаточно хорошие приближения алгебраическими числами. В 2000 г. В. Г. Чирский [4] рассмотрел обобщение понятия глобального соотношения на случай ряда, сходящегося в каждом из полей $\mathbb{K}_{v}$ к элементу этого поля, хорошо приближаемому числами из $\mathbb{K}$.

В настоящей работе получена теорема, дающая полностью эффективное решение задачи о глобальных соотношениях для вышеупомянутого обобщения понятия глобального соотношения.

Теорема. Пусть $f_{1}(z), \ldots, f_{m}(z) \in F\left(\mathbb{K}, c_{1}, c_{2}, c_{3}, q\right)$ составляют решение системы линейных дифференциальных уравнений

$$
y_{i}^{\prime}(z)=\sum_{j=1}^{m} Q_{j, i}(z) y_{j}(z), \quad i=1, \ldots, m, \quad Q_{j, i}(z) \in \mathbb{K}(z), \quad i, j=1, \ldots, m,
$$

и алгебраччески независимы над $\mathbb{K}(z)$. Пусть $\theta_{k}-$ целье числа из $\mathbb{K} u$

$$
\xi=\sum_{k=0}^{\infty} \theta_{k}, \quad \Theta_{n}=\sum_{i=1}^{n} \theta_{i}
$$

Пусть $\varepsilon>0,0<\delta<1, d \in \mathbb{N}, M=\left(\begin{array}{c}m+d \\ m\end{array}\right)$ и существует бесконечное множество номеров $n$ таких, что для всех простых чисел $p$ таких, что $p \leqslant \exp \left(\ln ^{1+2 \varepsilon}\left|\overline{\Theta_{n}}\right|\right), u$ любого нормирования $v$, продолжающего р-адическое нормирование в поле $\mathbb{K}$, выполнено неравенство

$$
\left|\xi-\Theta_{n}\right|_{v}<\exp \left(-(M-1+\delta) \exp \left(\ln ^{1+\varepsilon}\left|\overline{\Theta_{n}}\right|\right) \ln ^{1+2 \varepsilon}\left|\overline{\Theta_{n}}\right|\right) .
$$


Пусть последовательность $h_{n}$ определена равенством

$$
\ln h_{n}=\delta \exp \left(\ln ^{1+\varepsilon}\left|\overline{\Theta_{n}}\right|\right) \ln ^{1+\varepsilon}\left|\overline{\Theta_{n}}\right|-c_{4} \exp \left(\ln ^{1+\varepsilon}\left|\overline{\Theta_{n}}\right|\right) \ln ^{(1+\varepsilon) / 2}\left|\overline{\Theta_{n}}\right|,
$$

где $c_{4}=2+c_{5}$ nрu $\varepsilon \geqslant 1 u c_{4}=2$ npu $\varepsilon<1, a c_{5}=M^{2}\left(c_{1}^{*} \log _{p} q^{*}+1.25 c_{2}^{*} c_{3}^{*}+2 c_{3}^{*}+5\right)$.

Тогда существует эффективная постоянная $n_{0}$ такая, что для любого отличного от тождественного нуля многочлена $P\left(y_{1}, \ldots, y_{m}\right)$ степени $d$ по совокупности переменных с коэффициентами из $\mathbb{Z}_{\mathbb{K}}$, высота которого $h$ удовлетворяет условиям $h_{n-1}<h \leqslant h_{n}$ при $n>n_{0}$, существуют простое число $p$ в интервале

$$
\left|\overline{\Theta_{n}}\right| \leqslant p \leqslant M \exp \left(\ln ^{1+\varepsilon}\left|\overline{\Theta_{n}}\right|\right)
$$

u нормирование $v$, продолжающее р-адическое нормирование в поле $\mathbb{K}$, такие, что в поле $\mathbb{K}_{v}$

$$
\begin{aligned}
\left|P\left(f_{1}(\xi), \ldots, f_{m}(\xi)\right)\right|_{v} & \geqslant \exp \left(-\ln h+\left(-\frac{M-1}{\delta}-\frac{c_{4}(M-1)(1-\delta)}{\delta\left(\ln \ln h_{n}\right)^{\varepsilon /(1+\varepsilon)}}\right) \ln h_{n}\right) \\
& =\exp \left(-B\left(h, h_{n}\right)\right) .
\end{aligned}
$$

Для $n_{0}$ имеется оценка сверху через высоту $H(\mathbf{D})$ и степень $\tilde{d}$ системы $(1)$ :

$$
\begin{gathered}
n_{0} \leqslant(m+M)^{2 M} C(\kappa, m, \tilde{d}) H(\mathbf{D})^{c(\kappa, m, \tilde{d})}, \\
c(\kappa, m, \tilde{d})=\log _{2} C(\kappa, m, \tilde{d})=(2 \kappa(\tilde{d}+1) m)^{(2(\tilde{d}+1) m)^{8 m}} .
\end{gathered}
$$

Точные определения $H(\mathbf{D})$ и $\tilde{d}$ даны в [5; замечание 1.5$]$.

Наличие эффективной оценки снизу позволяет указать теоретически эффективный алгоритм для проверки, выполняется ли соотношение (2) в поле $\mathbb{K}_{v}, v \mid p, p \leqslant$ $M \exp \left(\ln ^{1+\varepsilon}\left|\overline{\Theta_{n}}\right|\right)$. Для этого достаточно рассматривать $N$-е частичные суммы рядов $f_{i}(\xi)$, где

$$
N \leqslant p^{2}(p-1) \frac{\kappa B\left(h, h_{n}\right)}{\kappa_{v} c_{3} \ln p}-1 .
$$

В качестве приложений теоремы рассматриваются гипергеометрические ряды следующего вида: $f(z)=\sum_{n=0}^{\infty} \frac{\left(\eta_{1}\right)_{n} \cdots\left(\eta_{r}\right)_{n}}{\left(\lambda_{1}\right)_{n} \cdots\left(\lambda_{s}\right)_{n}}(z t)^{t n}$, где $(\alpha)_{0}=1, \quad(\alpha)_{n}=\alpha(\alpha+1) \cdots$ $(\alpha+n-1), n \geqslant 1$. Для доказательства алгебраической независимости таких рядов использованы результаты В. Х. Салихова [7].

Теорема может быть применена к точкам $\xi$ вида

$$
\xi=\sum_{n=1}^{\infty} \prod_{k=1}^{n} p_{k}^{\gamma_{k}(n)}
$$

где $p_{k}-k$-е простое число, а $\gamma_{k}(n)$ - возрастающая последовательность натуральных чисел, удовлетворяющая некоторым условиям, которые мы ввиду их громоздкости опускаем. Другим примером служат ряды, рассмотренные в [4].

\section{Список литературы}

[1] А.Б. Шидловский, Трансцендентные числа, Наука, М., 1987. [2] Е. Bombieri, Recent progress in analytic number theory, vol. 2 (Durham, 1979), Academic Press, London, 1981, 1-68. [3] В. Г. Чирский, Матем. заметки, 48:2 (1990), 123-127. [4] В. Г. Чирский, Арифметические свойства рядов некоторых классов в полях с неархимедовыми нормированиями, Изд-во мех-мат. ф-та МГУ, М., 2000. [5] D. Bertrand, V. G. Chirskii, J. Yebbou, Ann. Fac. Sci. Toulouse Math. (6), 13:2 (2004), 241-260. [6] А. И. Галочкин, Вестн. Моск. ун-та. Сер. 1. матем., мех., 1970, №5, 58-63. [7] В. Х. Салихов, Докл. АН СССР, 307:2 (1989), 284-287.

T. P. Азаматов (T. R. Azamatov)

Московский государственный университет им. М. В. Ломоносова

E-mail: azamatov@mail.ru
Представлено А. В. Михалёвым Принято редколлегией 09.07.2007 